epigastrium, a bloated countenance, and a quick pulse. The chest was clear on percussion, and the respiratory murmur natural. The whistling in the larynx sufficed for the diagnosis, and tracheotomy was performed. During the performance of the operation the boy declared that he had coughed up the stone and swallowed it. The symptoms were relieved, and the whistling had ceased, but as the wound closed the distress and the whistling noise returned, proving that the stone had remained in the larynx, and that the noise had ceased only in consequence of air having been admitted below. Soon after, it was found to change its position and to pass down the right bronchus, again to be driven upwards into the larynx. By a second operation it was extracted, and the boy recovered without a bad symptom.

It has usually been observed that when the foreign body has become impacted in the larynx the symptoms from the first have been violent and distressing. The ordinary symptoms have been incessant spasmodic cough, croupy breathing, hoarseness, or complete aphonia, pain in the region of the larynx, and fits of suffocative dyspnœa. The termination of the case may be by sudden death, in consequence of closure of the glottis; or the foreign body may be expelled, or it may fall into the trachea. Then there may be an interval of comparative ease, succeeded either by a return of laryngeal symptoms, if the body is movable and light enough to be coughed upwards to the larynx, or, if it remains impacted in the bronchus, symptoms of inflammation of the lung may supervene.

An inspection of the widely open state of the glottis during deep inspiration shows that bodies of considerable size may easily pass between the vocal cords. A curious variety of substances have passed "the wrong way" and got into the bronchi. Thus the root and fangs of a tooth, an artificial tooth, a piece of bone in several instances, a cherry-stone and a plum-stone in several instances, a piece of flint, a button in two cases, a head of grass in two cases, an ear of rye, a Portugal ducat, a half-sovereign (Mr. Brunel's case), a sixpence, a shilling in two cases mentioned by Sir Thomas Watson, a piece of slate-pencil, a nut, a piece of nutshell, a piece of walnut-shell, ${ }^{4}$ a piece of nutmeg, a piece of cheese, a glass-bead, a leaden shot one-eighth of an inch in diameter, an iron nail, a metallic screw, a kidney-bean, \&c.

(To be concluded.)

NOTE ON THE

\section{TREATMENT OF ULCERS AND VARICOSE VEINS BY MARTIN'S STRONG ELASTIC BANDAGE.}

By GEORGE W. CALLENDER, F.R.S., SURGEON TO ST. BARTHOLOMEW'S HOSPITAL.

HAVING for some time used the elastic bandage as recommended by Dr. Martin, of Boston, U.S., for the treatment of varicose veins and ulcers, it is right to express my opinion that his treatment is practically good. Under its influence, ulcers not of a specific character do certainly heal, and quickly; and as this is effected whilst the patient follows his usual occupation, the merit of the treatment is evident. Although there has been scarcely time since I received the bandages from America for use in St. Bartholomew's Hospital $^{1}$ to test their efficacy in the continued treatment of varicose veins, yet I have found that the application of the bandage is at once followed by a sense of great relief. A man of thirty-six years of age, who had suffered for eleven years from varix of either internal saphenous vein, extending as high as the groin, experienced at once and since greater relief from the use of the bandage than he had ever had from silk elastic stockings, although his position was such as to allow of his wearing the best which could be made for him. I have used the bandages for many cases of vlcer of the leg, chiefly due to varicose veins, and always with the good result stated by Dr. Martin, with whose practical statement respecting the treatment of these ulcers I agree.

There are some points named by Dr. Martin which are

4 Dr. Gibb: Path. Trans, xiv., 41

1 The bandages are now supplied by Messrs. Krohne and Sesemann. essential if good results are to be had. The first of these is as to the quality of the bandage itself. It should be just such as the author describes, and as is supplied from America. If thicker, it would be clumsy; and if thinner, it needs to be more tightly drawn to offer the due resistance, and the edges are more apt to indent or cut into the flesh. If Dr. Martin's treatment is to be tried, the bandages should be such as his experience has led him to prefer.

The bandage should be applied before the patient rises from his bed in the morning-before, that is, the veins of the leg become distended by the impeded column of blood within them. It should be applied with just snugness enough not to slip down. The moment the foot is put to the ground the limb is so increased in bulk by the increase of blood in the veins that the bandage becomes of precisely the proper degree of tightness, and, no matter how active the exercise or labour of the patient, it will remain in position all day. When the patient undresses at night the bandage is to be removed, and the limb wiped dry, a piece of soft old linen moistened with oil, or some equally simple dressing, laid on the ulcer and retained in place by a few turns of an ordinary roller. The bandage should be sponged with water (cold will do, but warm is better), and hung over a line to dry in readiness for the morning, or it can be wiped dry at once, or rolled up with the tapes in the centre. Such is the dressing for the night; in the morning the leg can be washed, but, whether it is or not, all traces of oil or cerate should be carefully wiped away, as contact with the bandage of any fatty matter would tend gradually to injure the rubber. This is the whole treatment. Rubber bandage all day, with erect position and exercise. The simplest possible dressing (merely to protect the ulcer from injury), with the horizontal position and rest, all night. Any pimples which may form are left to be treated by the rubber, and if the skin become chafed a light covering, as of bunting, is applied as a bandage under the rubber, and the same is recommended to absorb excessive moisture.

No more distressing cases than of patients suffering from chronic ulcers crowd our hospitals, and I must say that I feel much indebted to Dr. Martin for the suggestion he has published, and which he has made practical application of for many years with, as he says, great success. My present but recent experience leads me to believe that he is to be confirmed in the statement he makes, and which any who like can read, as it is very clearly and ably put in the Transactions of the American Medical Association for 1877.

\section{A NEW OPERATION FOR THE CURE OF DETACHMENT OF THE RETINA.}

BY J. R. WOLFE, M.D., F.R.C.S.E. SURGEON TO THE GLASGOW OPHTHALMIC INSTITUTION, LECTURER ON OPHTHALMOLOGY IY ANDERSON'S UNIVERSITY.

THE effusion of serum between the retina and choroid, in an eye which is otherwise healthy, gives rise to the pathological condition called "detachment of the retina." This condition manifests itself to the patient first by dimness of vision; then by interruption of the field of vision-i.e., he sees when looking in one direction, but when trying to look in another a cloud seems to intervene between him and the object. Ophthalmoscopic examination by the mirror alone shows, in the first stage, a slight undulation of the retina. When further advanced a regular grey elevation becomes visible, which screens a part of the fundus when the eve is in motion. When examined more closely by the erect image, the retinal vessels are seen crossing its surface (partial detachment). This grey opaque body gradually increases in size, and the subretinal fluid accumulates until no part of the fundus can be seen. This stage coincides with total loss of vision, and the detachment is said to be complete.

This affection is still classed among the incurable diseases to which the eye is subject. All medical treatment by the administration of mercury, artificial leeching, \&c., has proved worse than useless. Von Graefe introduced an operation by puncturing the retina with a needle, and allowing the fluid to burst into the vitreous humour; and 